Objectives To investigate whether ankylosing spondylitis (AS) in women comprises a different clinical group compared to men. Methods Seventy-eight patients with AS were diagnosed and followed up since 1981 . We reevaluated all these patients by entering the demographic, clinical, laboratory, radiological and therapeutic disease characteristics in the database.

Results Seventy patients were identified as AS. Eight were excluded. Six developed psoriasis during follow up, one had Forestier?s disease and one developed Paget?s disease of bone. Sixty-one were men and 9 women. There were no differences between men and women concerning the age at disease onset. However, an infection had proceeded the diagnosis of AS in women $(\mathrm{p}<0.01)$. Axial and peripheral arthritis were similar between the two groups, although the elbows were more frequently involved in women $(p<0.05)$. Concerning systemic manifestations, no differences were found between the two groups. Finally, female patients had higher values of erythrocyte sedimentation rate (ESR) (47.5 [21.6]) compared to men (31.1 [23.0]) $(\mathrm{p}<0.05)$ and presented more frequently with anaemia of chronic disease (ACD) $(\mathrm{p}<0.01)$.

Conclusion AS in women is a rare condition (1:8). It seems that it is not a mild disease. The clinical picture is almost similar to men, but expresses high values of ESR and ACD.

\section{AB0132 FOUR CASES IN WHICH THE INITIAL ILLNESS WAS TYPICAL OF REITER'S SYNDROME AND LATER BECAME TYPICAL PSORIATIC ARTHRITIS}

${ }^{1} \mathrm{~K}$ Imre, ${ }^{1} \mathrm{E}$ Koo, ${ }^{2} \mathrm{M}$ Sesztak. ${ }^{1}$ Rheumatology; ${ }^{2}$ Rheumatology, Polyclinic of the Hospitaller Brothers of St. John of God in Budapest, Budapest, Hungary

\subsection{6/annrheumdis-2001.341}

Background It is generally accepted that in some patients reactive arthritis may progress further to ankylosing spondylitis. A generally accepted estimate is that 20 percent of patients with Reiter`s syndrome develop peripheral arthritis or axial disease. Objectives The study group consisted of 500 unrelated consecutive patients with psoriatic arthritis at 15 year follow up at the PsA outpatient clinic. We found four cases in which the initial illness was typical of Reiter`s syndrome and later became typical psoriatic arthritis.

\section{Methods}

Results One patient had persistent joint symptoms, two patients have at every 5 years recurrent bouts of arthritis and one patient suffered from severe, aggressive and unremitting disease.

Over 1 to 5 years the skin lesions progressively assume the characteristics of psoriasis.

The diagnosis of PsA was set up among 1-10 years.

Conclusion The possibility that psoriatic arthritis and Reiter`s syndrome may be similar or closely related is illustrated by our cases, too. Such cases have suggested a more than coincidental interrelationship between Reiter`s syndrome and psoriatic arthritis.

\section{AB0133 BLUE COLOURED SKIN IN PSORIATIC ARTHRITIS}

I Jajic, Z Jajiæ, T Nemcic. Department of Rheumatology, University Hospital ?Sestre Milosrdnice?, Zagreb, Croatia
Objectives In this paper, we have analysed blue coloured skin over involved joints as a one of the clinical features of the psoriatic arthritis that is helpful for simpler and earlier reaching the diagnosis.

Methods Clinical material consisted of 650 consecutively hospitalised and outdepartment patients with the diagnosis of PsA and 1265 patients with diagnosis of RA in period between 1975 and 1998 year. Special attention was attended to inspection of the skin over involved joint including skin appearance, colour and size of the involved area and its changes in different conditions of the weather and temperature.

Results We have found blue coloured skin over involved joints in 598 patients with PsA (92\%) and in 63 patients with RA (5\%) (N2 joints and digits in case of sausage digits. In less involved joints blue colour develops in minor area, usually on central dorsal part of the sin over the joint. Blue coloured skin was better visible in the cold winter days while in the summer season and during the remission of the disease as well as at room temperature, blue colour is less marked or even disappears.

Conclusion This sign is reliable, so it should be very useful in PsA diagnostics.

\section{AB0134 SULPHASALAZINE (SALAZOPYRINE EN) IN TREATMENT OF REACTIVE ARTHRITIS (EFFICACY, SAFETY AND TOLERABILITY)}

'Z Gnjidic, ${ }^{2} \mathrm{M}$ Gnjidic. 'Rheumatology, Institute of Rheumatology Dr. Drago Cop; ${ }^{2}$ Biology, University of Zagreb, Zagreb, Croatia

10.1136/annrheumdis-2001.343

\section{Background}

Objectives Evaluation of the efficacy and tolerance of sulphasalazine (SALAZOPYRIN EN-tabs) in the management of Reactive arthritis (ReA) triggered by a urogenital infection.

Methods We conducted an open randomised trial on 2 parallel groups of patients, 30 patients each during 12 months (30 treated with SALAZOPYRIN EN 1-2 g/day, versus 30 treated with NSAID or analgesics). Tolerability and safety were assessed with whole blood picture test for liver and renal function, gastro-intestinal (G-I) adverse effects, and other side effects. Every month the efficiency of treatment was checked by clinical and laboratory methods.

Results There were no significant differences in demographic, clinical and laboratory parameters among the two groups at the study entry. By the end of the study, there was evidence of significant improvement shown by pain score, ability of movement, and reduction in the acute phase response in sulphasalazine (SALAZOPYRINE EN) group. Adverse reactions in the sulphasalazine (SALAZOPYRINE EN) group (12\% of patients) occurred mainly in the first ten weeks of treatment and were mild, reversible and did not require termination of therapy. In $1 \%$ of patients these were severe enough to stop treatment.

The NSAID group encountered adverse reactions in $27 \%$ of patients and $4 \%$ required termination of therapy.

Conclusion Sulphasalazine (SALAZOPYRINE EN) proved to be a good first choice as a second-line of a disease modifying antirheumatic drug because of its early efficacy ( 8 weeks), persistent efficacy and its good tolerance. 


\section{AB0135 MEASURING ANKYLOSING SPONDYLITIS: IS IT USEFUL? PRELIMINARY RESULTS}

M Cruz, C Reis, A Alves de Matos, JC Branco. Unidade de Reumatologia, Hospital de Egas Moniz, Lisboa, Portugal

10.1136/annrheumdis-2001.344

Background Ankylosing spondylitis is a potentially limitating rheumatic disease, moreover because it affects adolescents and young adults. Sometimes it can be incapacitating, not only because of its spondylitic features but also because of the associated complications and extra-spondylarthropathy manifestations. It is a well known association with the histocompatibility antigen HLA B27, but the exact nature of this association remains hypothetic.

Objectives To apply disease indexes and measures to our Unit's patients population and correlate them with the presence of HLA B27. To apply a psychometric test to this population and correlate the results with some parameters of disease measurement.

Methods We describe and characterise our Unit's ankylosing spondylitis patients population in terms of demographis parameters and presence of HLA B27. In a single visit we measured some disease indexes (Schober value, Mander index, BASFI and BASDAI indexes) and applied the Scl-90 questionnaire.

Results From our baseline population of 34 patients, 14 were evaluated until now with these parameters. We found 5 women (36\%) and 9 men (64\%), with mean age 49,8 \pm 7 SD years old and 47,8 $\pm 15,4$ SD years old, respectively. Almost all patients ( $\mathrm{n}=12,86 \%$ ) were white, being one yellow and one black. Mean age at diagnostic was 43,6 \pm 7,8 SD years old for women and $32,9 \pm 12,2$ SD years old for men, which seems to confirm the more benign course in women. Ten $(71,4 \%)$ of these patients had extra-spondylarthropathy manifestations. In eight (57\%) HLA B27 was positive, in four $(28,6 \%)$ it was unknown and in two $(14,3 \%)$ it was negative. Six patients had a Schober value between 0 and $1 \mathrm{~cm}$, five between 1,5 and $3 \mathrm{~cm}$ and three between 3,5 and $4,5 \mathrm{~cm}$. From this last group, two were thw ones with negative HLA B27. Mander index was between 0 and 10 in nine patients, between 11 and 20 in three and between 21 and 30 in two. Only 13 from the 14 patients answered the BASFI and BASDAI indexes and Scl-90 questionnaire. In six patients BASFI score was between 0 and 3 , in five between 3,1 and 5 and in two between 5,1 and 8. BASDAI score was between 0 and 3 in four patients, between 3,1 and 5 in six and between 5,1 and 8 in three. Scl-90 test revealed psychologic pathology in eight of thirteen patients: four in the range of obsessive-compulsive, four with interpersonal sensitivity, three with somatization, three with hostility, two with paranoid ideas and one with psycoticism. We will evaluate the rest of the patients to finish this study.

Conclusion As is commonly described, we found in our ankylosing spondylitis population a majority of males, in a 2:1 rate; age at diagnosis was more advanced in women, probably due to a more benign and/or slow progression of disease. Patients without HLA B27 antigen had better Schober measures, probably signifying a more benign disease. The majority of patients revealed different types of psychopathology by Scl 90, which may be associated with bad coping with the disease.

\section{AB0136 AORTIC INVOLVEMENT IN ANKYLOSING SPONDYLITIS}

J Hrba. Institute of Rheumatology, Prague, Czech Republic

\subsection{6/annrheumdis-2001.345}

Background In ankylosing spondylitis (AS) the occurrence of involvement of ascending aorta and aortic (and sometimes mitral) valve is known. The microscopic changes include inflammatory destruction of aortic and valvular tissue and replacement by granulation and fibrous tissue. Aortic regurgitation results from thickening and displacement of valve cusps and dilatation of aortic root. It may be clinically silent or may cause severe problems to the patient. Immunosuppressive therapy may prevent or delay the need for aortic valve replacement but many times valve replacement is the only life-saving therapy.

Objectives

Methods

Results Recently we had an opportunity to treat 3 patients with AS and aortic incompetence. All three were suffering from AS with peripheral arthritis. In one patient aortic failure was complicated with bacterial endocarditis although previous symptoms of aortic failure were only subclinical. In the second patient aortic insufficiency proceeded very rapidly to severe and lifethreatening cardiac failure. In both of them aortic valve replacement was successful. Interestingly, in the second patient massive immunosuppressive therapy prior to cardiosurgery led to a significant improvement of cardiac failure. The third patient has not been suffering from any cardiac troubles but the clinical and x-ray examination (aortography) revealed a significant aortic regurgitation.

Conclusion It is a matter of interest that the second patient had some features of Marfan's syndrome and the third had some features of Ehlers-Danlos syndrome. It raised the question which of these two components ? inflammation or primary defect of collagen or both of them participates in the aortic involvement.

\section{OP0054 COST-EFFECTIVENESS OF COMBINED SPA-EXERCISE THERAPY IN ANKYLOSING SPONDYLITIS: A RANDOMISED CONTROLLED TRIAL}

${ }^{1} \mathrm{~A}$ Van Tubergen, ${ }^{1} \mathrm{~A}$ Boonen, ${ }^{1} \mathrm{R}$ Landewé, ${ }^{2} \mathrm{M}$ Rutten-van Mölken, ${ }^{1} \mathrm{D}$ Van der Heijde, ${ }^{3}$ A Hidding, ${ }^{1}$ SJ Van der Linden. ${ }^{1}$ Rheumatology, University Hospital Maastricht, Maastricht; ${ }^{2}$ Technology Assessment, Erasmus University, Rotterdam; ${ }^{3}$ Institute for Rehabilitation Research, Hoensbroek, Netherlands

10.1136/annrheumdis-2001.346

\section{Background}

Objectives To evaluate the incremental cost-effectiveness and cost-utility of a 3 weeks course of combined spa-exercise therapy followed by weekly group physical therapy, compared to weekly group physical therapy alone in ankylosing spondylitis (AS) patients.

Methods 120 Dutch AS outpatients were randomly allocated into three groups of 40 patients each. Group 1 was treated at a spa resort in Bad Hofgastein, Austria; group 2 at a spa resort in Arcen, The Netherlands. A control group stayed at home and continued weekly group physical therapy. Spa-exercise therapy consisted of physical exercises, walking, postural correction therapy, hydrotherapy, sports and either visits to the so-called Gasteiner Heilstollen (Austria) or the sauna (The Netherlands). After spa-exercise therapy all patients followed weekly group physical therapy for another 37 weeks. Effectiveness of the intervention was assessed by functional ability (BASFI) (range 